\title{
Effects of 3,5-diiodo-L-thyronine on the liver of high fat diet fed rats
}

\author{
Marco Giammanco, ${ }^{1}$ Stefania Aiello, ${ }^{1}$ Alessandra Casuccio, ${ }^{2}$ Maurizio La Guardia, ${ }^{3}$ Luca Cicero, ${ }^{4}$ \\ Roberto Puleio, ${ }^{4}$ Irene Vazzana, ${ }^{4}$ Giovanni Tomasello, ${ }^{1}$ Giovanni Cassata, ${ }^{4}$ Gaetano Leto, ${ }^{2}$ \\ Danila Di Majo' \\ ${ }^{1}$ Department of Experimental Biomedicine and Clinical Neurosciences, University of Palermo, \\ Palermo; ${ }^{2}$ Department of Sciences for Health Promotion and Mother-Child Care, University of \\ Palermo, Palermo; ${ }^{3}$ Department of Biological, Chemical and Pharmaceutical Sciences and \\ Technologies, University of Palermo, Palermo; ${ }^{4}$ Institute for Experimental Veterinary Medicine of \\ Sicily, Palermo, Italy
}

\begin{abstract}
Experimental studies have highlighted that the administration of 3,5-diiodo-L-thyronine (T2) to rats fed diets rich in lipids induces a decrease of cholesterol and triglycerides plasma levels and body weight (BW) without inducing liver steatosis. On the basis of these observations we carried out some experimental in vivo studies to assess the effects of multiple high doses of T2 on the pituitary thyroid axis of rats fed diet rich in lipids. Fifteen male Wistar rats were divided into three groups of five animals each. The first group (N group) received standard diet, the second group was fed with a high fat diet (HFD group), while the third group (HFDT2 group) was additionally given T2 intraperitoneally at a dose level of $70 \mu \mathrm{g} / 100 \mathrm{~g}$ of BW three times a week up to four weeks. At the end of the treatment, blood sample from each animal was collected, centrifuged and the serum was stored at $-20^{\circ} \mathrm{C}$. The serum concentrations of thyroidstimulating hormone (TSH), triiodothyronine, thyroxine, adrenocorticotropic hormone, triglycerides, cholesterol, glucose, alanine
\end{abstract}

Correspondence: Marco Giammanco, Department of Experimental Biomedicine and Clinical Neurosciences - BioNec, University of Palermo, Palermo, Italy.

Tel: $+39.091 .6555805 ;+39.333 .4706470$.

E-mail: marco.giammanco@unipa.it

Key words: 3,5-diiodo-L-thyronine; TSH; Thyroid hormone; Hepatic steatosis.

Conflict of interest: the authors declare no potential conflict of interest.

Received for publication: 4 December 2015.

Accepted for publication: 28 December 2015.

(C) Copyright M. Giammanco et al., 2016

Licensee PAGEPress, Italy

Journal of Biological Research 2016; 89:5667

doi:10.4081/jbr.2016.5667

This article is distributed under the terms of the Creative Commons Attribution Noncommercial License (by-nc 4.0) which permits any noncommercial use, distribution, and reproduction in any medium, provided the original author(s) and source are credited. aminotransferase, aspartate aminotransferase, alkaline phosphatase were then determined. In addition, liver of rats was examined by histology in order to assess the presence and degree of steatosis. The administration of T2 to rats fed with a high fat diet suppressed TSH secretion $(\mathrm{P}=0.013)$ while no steatosis was observed in the liver of these animals. Our data show that multiple administrations of high doses of T2 to rats fed diets rich in lipid inhibit TSH secretion and prevent the onset of liver steatosis in these animals.

\section{Introduction}

Thyroid hormones thyroxine (T4) and triiodothyronine (T3) are well known to stimulate energy metabolism in both animals and humans. ${ }^{1,2}$ This phenomenon is mainly mediated by T3 that is considered to be the main active molecule. On the basis of these findings several studies have been undertaken to investigate the possible clinical use of this hormone in the treatment of diseases that are associated with an over consumption of food and drinks high in fat and/or sugar such as obesity, diabetes, dyslipidemia and hepatic steatosis. In particular, T3 has long been considered potentially suitable for the treatment of obese patients as it has been shown to induce a decrease of the body weight (BW) following stimulation of lipid catabolism and a daily increase of energy expenditure. However, experimental in vivo studies report that the administration to rodents of 3,5-diiodo-L-thyronine (T2), which has long been considered only an inactive metabolite of T3 and T4, increased their resting metabolic rate (RMR). ${ }^{3,4}$ On the other hand, some studies in humans have confirmed that the administration of T2 can increase the basal metabolic rate and decrease fat and BW without side effects. ${ }^{3}$ On the basis of these observations, several $\mathrm{T} 2$ analogs have been recently designed and synthesized with the aim of finding novel and more effective pharmacological approaches in the treatment of these pathological conditions. In this context, the administration of the analog TRC150094 to rats fed a high fat diet resulted in reducing the accumulation of fat in the liver and adipose tissue and in decreasing cholesterol and triglycerides blood levels without suppressing pituitary thyroid-stimulating hormone (TSH) levels. ${ }^{5}$ On the basis of these observations we have performed some in vivo study to better define the effects of multiple administration of high doses of T2 on the onset of liver steatosis in rats fed diet rich in lipids and the influence of this treatment on TSH secretion as it has been earlier reported by Padron and colleauges. ${ }^{6}$ 


\section{Materials and Methods}

\section{Chemicals}

3,5-diiodo-L-thyronine was obtained from Sigma-Aldrich (St. Louis, M0, USA). An inclusion complex of T2 with hydroxypropyl cyclodextrin (HP-Cy) (Sigma-Aldrich) was produced to improve drug solubility and stability in PBS at pH 7.4. Appropriate amounts of T2 were suspended in a solution of cyclodextrin in redistilled water to obtain a final weight ratio HPCy/T2 of 4:1. Then, few drops of $0.1 \mathrm{~N}$ sodium hydroxide were added until the complete dissolution of the drug and the formation of inclusion complex. The $\mathrm{pH}$ was adjusted to 7 with of $0.1 \mathrm{~N}$ hydrochloric acid and the solution was freeze-dried. Appropriate amount of solid complex was weighed and injected to animals just before drug administration.

\section{Animals and treatments}

Male Wistar rats aged between 8 and 10 weeks and weighing 300 $350 \mathrm{~g}$, were purchased from Harlan Italy [S. Pietro al Natisone (UD), Italy], and kept at $24^{\circ} \mathrm{C}$ with a light/dark cycle of 12:12-h. Animals had free access to water and food. The maintenance and care of the animals were carried out according to the guidelines of the Council of the European Community for the care and use of animals. After 1 week of acclimatization, 15 rats were randomly divided into three groups of 5 rats each. The first group (N) received standard diet (total percentage of metabolizable energy: $60.4 \%$ carbohydrates, $29 \%$ protein, $10.6 \%$ fat, $15.88 \mathrm{~kJ}$ of energy/g); the second group was fed a high-fat diet (HFD) (percent of total metabolizable energy: 21\% carbohydrates, $29 \%$ protein, $50 \%$ fat, $19.85 \mathrm{~kJ}$ of energy $/ \mathrm{g}$ ); the third group (HFDT2) was fed as the previous one. Additionally, these ani- mals received intraperitoneal injections (ip) of T2 (70 $\mu \mathrm{g} / 100 \mathrm{~g} \mathrm{BW})$ three days a week, after anesthesia with inhaled isoflurane. Control rats (group $\mathrm{N}$ and group HFD) were injected with saline, after anesthesia with inhaled isoflurane. Body weight and food intake of each animal were recorded every two days. Food intake was not significantly influenced by the composition of the diet or treatment with T2. After 28 days of treatment, rats were anesthetized with inhaled isoflurane and intramuscular administration of tiletamine, $60 \mu \mathrm{L}$ of zolazepam, and $60 \mu \mathrm{L}$ of medetomidine, and killed by cervical dislocation. Their livers were rapidly dissected, weighed, cut into small pieces and quickly frozen in liquid nitrogen and stored at $-80^{\circ} \mathrm{C}$.

\section{Serum measurements}

Blood samples obtained by intracardiac puncture were collected and centrifuged. Serum was stored at $-80^{\circ} \mathrm{C}$ until assays. Serum concentrations of TSH third generation, T3, T4, adrenocorticotropic hormone $(\mathrm{ACTH})$, triglycerides, cholesterol, glucose, glutamic oxaloacetic transaminase (GOT), glutamic pyruvic transaminase (GPT), alkaline phosphatase (AP) were measured using a clinical analyzer Siemens Immunolite 2000 (Siemens Healtcare, Erlangen, Germany) following standard procedures.

\section{Histological analysis}

Liver tissue sections $3 \mu \mathrm{m}$ thick were fixed in $10 \%$ buffered formalin and stained with hematoxylin-eosin. The histological sections were then subjected to a semi-quantitative examination, to detect the percentage of hepatocytes showing macro or microvesicular steatosis $(>15 \mu \mathrm{m})$. The score system used to assess steatosis was defined as: i) absent or minimal when the histological lesions involved were $<1 \%$ of hepatocytes (score 0); ii) slight if $<30 \%$ of hepatocytes (score 1);

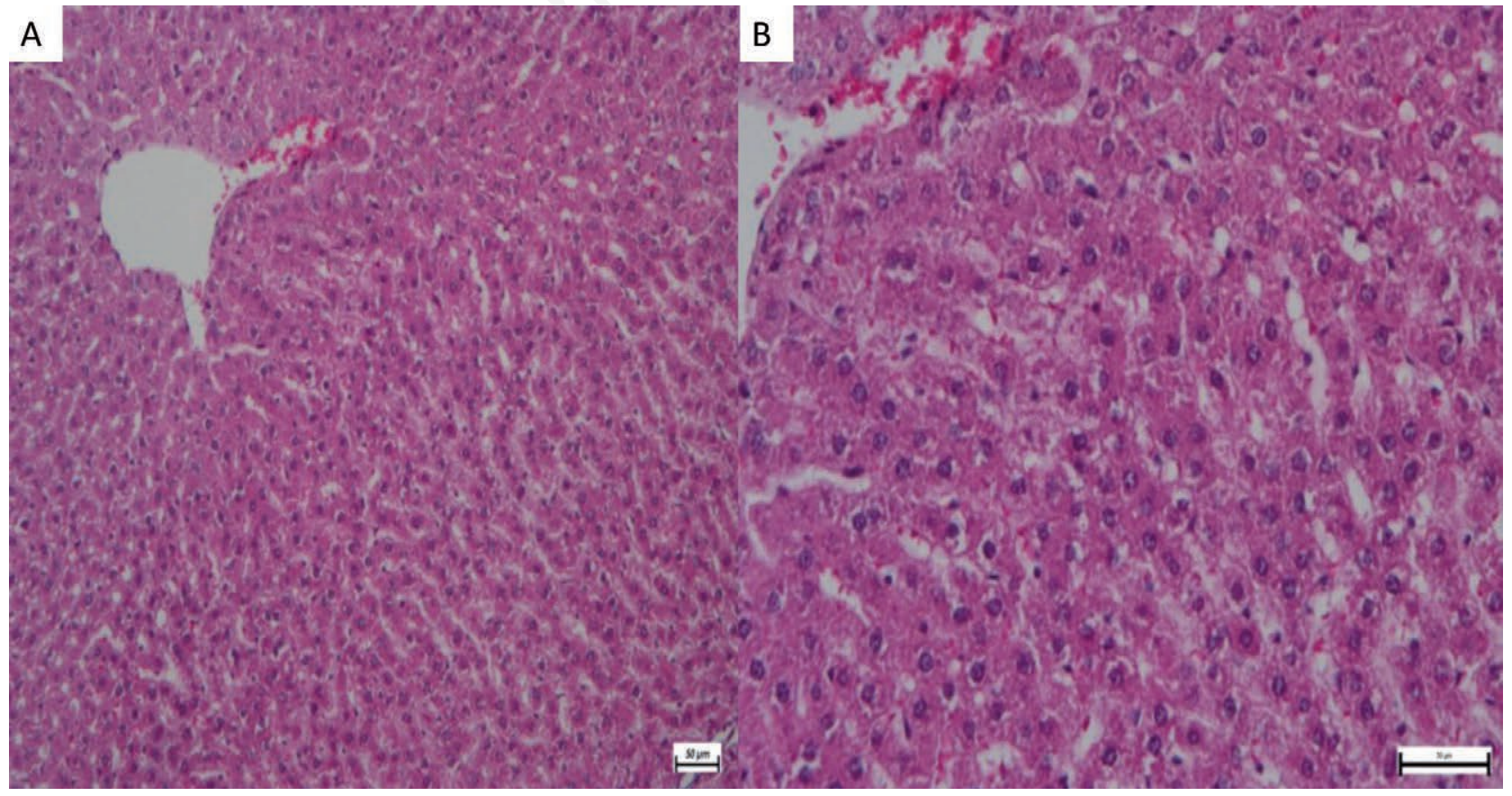

Figure 1. Histological section of liver tissue from control rats: no sign of steatosis is evident at either magnification [100× $(A)$ nor $200 \times(B)]$ (score 0). 
iii) moderate if the number of the hepatocytes involved were between 30 and $60 \%$ (score 2); and iv) severe if the hepatocytes involved were more than $60 \%$ (score 3 ). The percentage of hepatocytes involved was determined by counting cells in six microscopic fields at $400 \times$ magnification. Microscopic examination was carried out with Leica DMLB (Leica, Nussloch, Germany), equipped with Nikon (Tokyo, Japan); the system of image acquisition used was Nis elements Br software.

\section{Statistical analysis}

Continuous variables are expressed as mean \pm standard deviation. Intergroup differences among groups at $\mathrm{T} 0$ and $\mathrm{T} 28$ were assessed by the univariate analysis of variance (ANOVA), and post-hoc analysis with the Tukey's test was used to determine pairwise differences. The intragroup difference between different times was evaluated with the

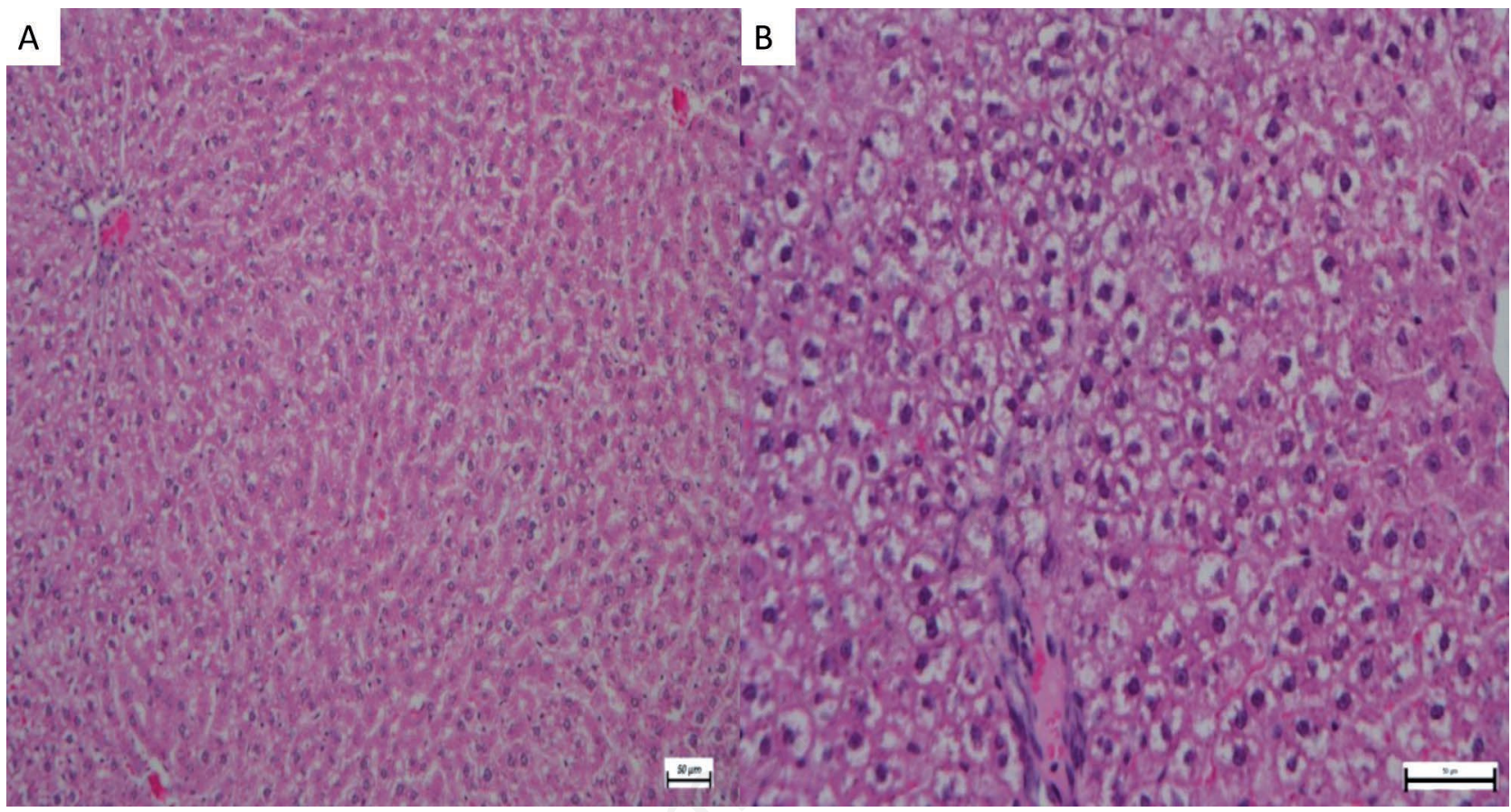

Figure 2. Histological sections of liver tissue from high fat diet fed rats. Note the widespread intracellular vacuolization of hepatocytes and the resulting relocation of cell nuclei in a peripheral position (score 2). A) 100× magnification; B) $200 \times$ magnification.

Table 1. Weight, metabolic and hormonal data of three groups.

\begin{tabular}{|c|c|c|c|c|c|c|}
\hline & \multicolumn{2}{|c|}{ HFD } & \multicolumn{2}{|c|}{ HFDT2 } & \multicolumn{2}{|c|}{$\mathbf{N}$} \\
\hline & T0 & T28 & TO & T28 & T0 & T28 \\
\hline \multirow[t]{2}{*}{ Weight } & $351.0(31.1)$ & $378.6(34.3)$ & $347.0(14.8)$ & $392.8(20.4)$ & $360.0(11.2)$ & $409.8(22.2)$ \\
\hline & \multicolumn{2}{|c|}{$\mathrm{P}=0.023^{*}$} & \multicolumn{2}{|c|}{$\mathrm{P}=<0.0005^{*}$} & \multicolumn{2}{|c|}{$\mathrm{P}=0.018^{*}$} \\
\hline \multirow[t]{2}{*}{ ACTH } & $111.1(79.6)$ & $55.1(9.2)$ & $39.8(17.2)$ & $44.0(15.6)$ & $99.6(42.2)$ & $44.3(17.4)$ \\
\hline & \multicolumn{2}{|c|}{$\mathrm{P}=0.222^{*}$} & \multicolumn{2}{|c|}{$\mathrm{P}=0.729 *$} & \multicolumn{2}{|c|}{$\mathrm{P}=0.033^{*}$} \\
\hline \multirow[t]{2}{*}{$\mathrm{T} 3$} & $121.0(9.6)$ & $55.1(9.2)$ & $115.0(27.5)$ & $44.0(15.6)$ & $116.3(21.7)$ & $44.3(17.4)$ \\
\hline & \multicolumn{2}{|c|}{$\mathrm{P}=0.009 *$} & \multicolumn{2}{|c|}{$\mathrm{P}=0.005^{*}$} & \multicolumn{2}{|c|}{$\mathrm{P}=0.004 *$} \\
\hline \multirow[t]{2}{*}{ TSH } & $0.31(0.09)$ & $0.20(0.11)$ & $0.61(0.51)$ & $0.06(0.05)$ & $0.72(0.79)$ & $0.65(0.46)$ \\
\hline & \multicolumn{2}{|c|}{$\mathrm{P}=0.049 *$} & \multicolumn{2}{|c|}{$\mathrm{P}=0.043^{*}$} & \multicolumn{2}{|c|}{$\mathrm{P}=0.814^{*}$} \\
\hline \multirow[t]{2}{*}{$\mathrm{T} 4$} & $6.7(0.5)$ & $3.6(0.4)$ & $5.7(2.2)$ & $3.2(1.2)$ & $7.3(1.8)$ & $4.9(1.5)$ \\
\hline & \multicolumn{2}{|c|}{$\mathrm{P}=0.004^{*}$} & \multicolumn{2}{|c|}{$\mathrm{P}=0.043^{*}$} & \multicolumn{2}{|c|}{$\mathrm{P}=0.116^{*}$} \\
\hline Glucose & - & $182.6(40.9)$ & - & $178.2(35.1)$ & - & $204.8(63.9)$ \\
\hline Cholesterol & - & $63.2(8.7)$ & - & $69.0(9.9)$ & - & $60.0(5.1)$ \\
\hline Triglycerides & - & $179.0(82.1)$ & - & 119.4 (35.7) & - & $172.8(75.0)$ \\
\hline GOT & - & 113.6 (23.7) & - & $89.8(12.5)$ & - & $98.6(13.2)$ \\
\hline GPT & - & $51.6(22.7)$ & - & 47.4 (11.7) & - & $32.6(4.9)$ \\
\hline $\mathrm{AP}$ & - & $171.4(48.7)$ & - & $176.8(59.8)$ & - & $102.4(13.6)$ \\
\hline
\end{tabular}

HFD, hight fat diet group; HFDT2, fat diet group treated with T2; N, control group; T0, day 0 of the test; T28, day 28 of the test; ACTH, adrenocorticotropic hormone; T3, triiodothyronine; TSH, thyroid-stimulating hormone; T4, thyroxine; GOT, glutamic oxaloacetic transaminase; GPT, glutamic pyruvic transaminase; AP, alkaline phosphatase. Values are expressed as mean (standard deviation). *T28 vs T0. 
paired sample Student's t-test. Data were analyzed by IBM SPSS Software 22 version (IBM Corp., Armonk, NY, USA). All P values were two-sided and $\mathrm{P}<0.05$ was considered statistically significant.

\section{Results}

The BW of the animals was recorded at the beginning of the treatment (day 0) and on day 28. No significant differences in the mean BW among the three groups of animals were observed (Table 1). However, a significant increase in BW was highlighted for each rat between day 0 and day 28 (Table 1). Furthermore, no significant differences in the serum levels of glucose or cholesterol were noted among the three groups on day 28 when compared to those measured on day 0 (Table 1). Still, the serum levels of triglycerides decreased in HFDT2 animals as compared to the other groups; this reduction did not reach the statistical significance. Among the biochemical parameters of liver function examined, only the plasma levels of AP resulted significantly more elevated $(\mathrm{P}=0.041)$ in the HFD and HFDT2 grous as compared to control group on day 28 while, again, no significant difference was observed for glutamic oxaloacetic transaminase and glutamic pyruvic transaminase. Interestingly, the mean plasma levels of $\mathrm{T} 3$ resulted in all the three groups significantly lower on day 28 than those measured on day 0 (Table 1). On the other hand, a similar trend was observed in the case of T4 plasma levels (Table 1). However, in this case, stastistically significant difference was observed only for the HFD group $(\mathrm{P}=0.004)$. Finally, no significant difference in the plasma levels of T3 and T4 was highlight among the three groups of animals between day 0 and day 28 . Although no significant difference in TSH plasma concentrations was observed among the different groups of animals, in the HFDT2 groups the circulating levels of this parameter on day 28 were significantly lower as com- pared to control group $(\mathrm{P}=0.013)$. In all the animals TSH levels were lower on day 28 as compared to day 0 . However these differences resulted statistically significant for the HFD group $(\mathrm{P}=0.049)$ e HFDT2 group $(\mathrm{P}=0.043)$. At the histological evaluation the rats of group $\mathrm{B}$ (standard diet) showed no signs of steatosis (Figure 1), while rats of the HFD group (high fat diet) showed a fatty medium entities (Figure 2), which was not highlighted in the control group and in HFDT2 group (Figures 1 and 3 ).

The histological sections of the liver of the animals of the group HFD, examined at $100 \times 200 \times$ magnification showed the presence of optically empty vacuoles in the cytoplasm of hepatocytes. In some cases this phenomenon determined the relocation of the nuclei in a peripheral position. Although cell damage was small, it was widespread and classified as fatty liver score 2 .

\section{Discussion}

Recent studies have shown the beneficial effects of 3,5-T2 in preventing liver steatosis in animals fed a high-fat diet. Experimental evidence indicates that the beneficial effects exerted by $3,5-\mathrm{T} 2$ are nongenomic but rather mediated by metabolic pathways mainly at the mitochondrial level. Our investigations were aimed at assessing the suppressive effects of this molecule on the pituitary-thyroid axis. ${ }^{7}$ In line with the recent findings of Padron and colleagues, ${ }^{6}$ our data show that the administration of 3,5-diiodo-L-thyronine to rats three times a week up to 4 weeks leads to a reduction in BW gain despite the decreased level of T4 and T3 serum levels. On the other hand, Lanni and colleagues ${ }^{8}$ have shown that the administration of $25 \mu \mathrm{g} / 100 \mathrm{~g}$ of $3,5-\mathrm{T} 2$ to rats may reduce $\mathrm{BW}$ gain and adiposity while no change in serum T4 levels was observed in animals fed a diet rich in fat and treat-

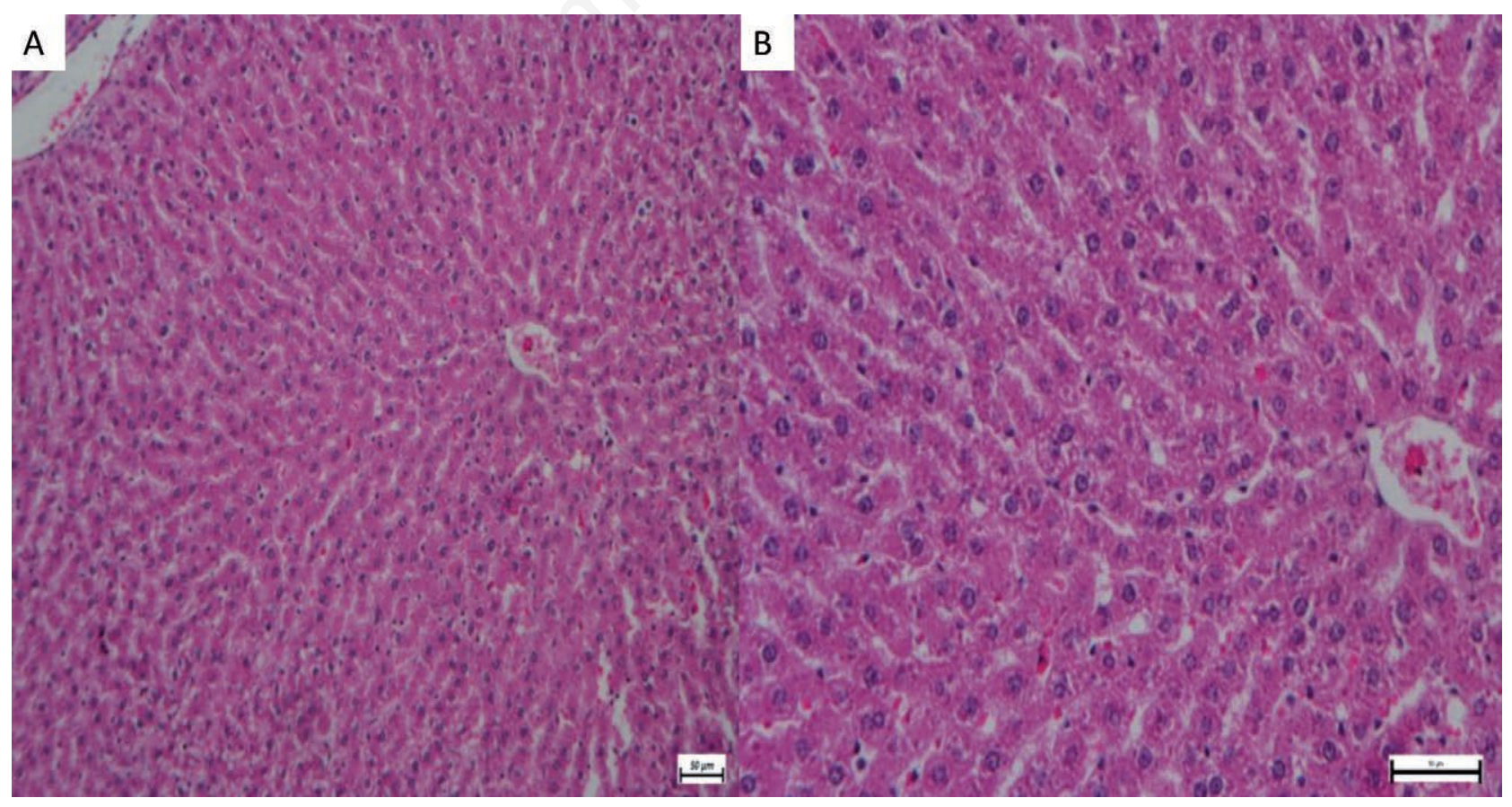

Figure 3. Histological section of liver tissue from 3,5-diiodo-L-thyronine high fat diet fed rats. No sign of steatosis is present (score 0). A) $100 \times$ magnification; B) $200 \times$ magnification. 
ed with 3,5-T2. This phenomenon was not observed in control animals. In addition, it was also shown that 3,5-T2 increased the rate of fatty acid oxidation in skeletal muscle. In addition to the chronic effects of 3,5-T2, a non-genomic acute and most likely increased mitochondrial activity in rat liver has also been described. ${ }^{2}$ An increased consumption of hepatic oxygen and oxidative activity in the liver of rats fed a highfat diet, and the recovery of energy expenditure in hypothyroid rats have been also described. ${ }^{9-11}$ The metabolic effects induced by 3,5-T2 could, in part, explain the decrease in BW gain and in that of retroperitoneal fat observed in aged rats treated with this agent. In addition to the beneficial effects of 3,5-T2 on BW increase and adiposity, rats treated with 3,5-T2 also showed an improvement in glucose tolerance, more than 10\%, compared to control animals. This result suggests that 3,5 T2 appears to improve glucose tolerance, directly or indirectly by decreasing adiposity in the treated animals. On the other hand, these findings are consistent with the observation that 3,5 -T2, in addition to other effects on the liver, prevents insulin resistance in skeletal muscle of rats fed a high-fat diet and increases the expression of GLUT4 by insulin-induced phosphorylation of Akt. 3,5-T2 also was shown to increase nuclear sirtuin 1 expression and to decreases lipogenic genes. ${ }^{12,13}$ In agreement with previous data demonstrating that 3,5-T2 exerts important metabolic effects, ${ }^{2}$ experimental findings highlight a significant increase in oxygen consumption in rats treated with 50 $\mu \mathrm{g} / 100 \mathrm{~g}$ of 3,5-T2 as compared to controls. Interestingly these animals had lower, serum levels of T3 and T4. These hormones are the main regulators of energy metabolism. Accordingly, 3,5-T2 may increase the rate of mitochondrial fatty acid oxidation and thermogenesis in the rat skeletal muscle, $\beta$-oxidation of lipids, ${ }^{8}$ mitochondrial oxygen consumption ${ }^{10}$ and also the RMR. ${ }^{14}$ Thus, it is conceivable to hypothesize that 3,5-T2 may function as a stimulator of RMR and may increase oxygen consumption. TSH serum levels were low. In animals treated with 3,5T2 this phenomenon did not appear to be related to the reduced serum levels of T4 and T3. However, Antonelli and colleagues ${ }^{3}$ have recently highlighted evident changes in the levels of thyroid hormones in serum in two euthyroid subjects treated for 3 weeks with a daily dose of 300 $\mu \mathrm{g}$ of 3,5-T2. On the other hand, Horst and colleagues ${ }^{9}$ and Giammanco and colleagues ${ }^{15}$ have shown that 3,5 -T2 reduces the secretion of TSH from the pituitary fragments of rat stimulated by T2. ${ }^{15}$ These authors also showed a reduction in T4 serum levels after 90 days of treatment with $25 \mu \mathrm{g}$ of 3,5-T2/100 g, these data are consistent with the results obtained by our studies. ${ }^{15}$ Furthermore, other observations highlighted that a single dose of 3,5-T2 reduced serum concentrations of the $\beta$ subunit of pituitary TSH. ${ }^{16}$ Thyroid hormone receptor $\beta$ (TR $\beta$ ) is the main mediator of the negative feedback of thyroid hormone. ${ }^{17}$ The results of Ball and colleagues ${ }^{16}$ showing that TR $\beta 2$ binds 3,5-T2 with higher affinity than the other TR isoforms may well explain the effectiveness of 3,5T2 in suppressing the secretion of TSH. Recently, it has been also reported that in humans that 3,5-T2 binds and activates the B isoform of human TR $\beta$, showing once again that this metabolite exerts genomic effects. ${ }^{18}$ Consequently the reduced serum levels of TSH in rats treated with 3,5-T2 may explain the reduction in their thyroid deiodinase D1. On the other hand, the activity of deiodinase D2 is increased both in the hypothalamus in the pituitary of rats treated with the 3,5-T2, despite the thyreomimetic effects of 3,5-T2. It has been shown that levels of D2 protein and its activities are regulated by post-translational mechanisms, in particular by ubiquitination processes, that seem to be mainly driven by T4. ${ }^{19}$ Since T4 levels were decreased in rats treated with 3,5-T2, this could explain the increase in Q2 in these animals. ${ }^{19}$ Taken together, the results show that the 3,5-T2 significantly down regulated thyroid function. In addition to the decreased levels of thyroid hormones, the inhibitory effect of 3,5-T2 on thyroid function appears, at least partly, to be due to reduced serum levels of TSH because it is the main stimulant of the thyroid gland. The expression of the TSH receptor (TSHR) and TSHR mRNA were increased in the thyroid of rats treated with 3,5-T2. It has been reported that TSH exerts inhibitory effects on the activity of the promoter of the gene for TSHR. ${ }^{20}$ Therefore, it is likely that thyroid TSHR expression is higher in rats treated with the 3,5-T2 as TSH serum levels were significantly reduced. Furthermore, the expression of NOX4 is inhibited, while that of DUOX2 is up-regulated, just as previously shown in other models. ${ }^{21,22}$ The chronic administration of 3,5-T2 reduces the increase in BW and retroperitoneal fat mass and increases RMR. These effects do not appear to be correlated with the decreased levels of thyroid hormone. The reduction in thyroid hormone levels may be secondary to the reduction in serum levels of TSH, which leads to a reduced activity and expression of sodium-iodide symporter (NIS), thyroid D1, and thyroid peroxidase (TP0). ${ }^{6}$ These new data support the hypothesis that 3,5 -T2 causes exogenous TSH suppression. Thus, 3,5-T2 may be used as a non-thyreomimetic pharmacological agent in the treatment of hypothyroidism. On the other hand, in hyperthyroidism, TSH circulating levels are typically $<0.1 \mathrm{mU} / \mathrm{L}$ or even lower than $0.05 \mathrm{mU} / \mathrm{L}$.

\section{Conclusions}

Until recently 3,5-T2 has been considered as a hormone with little or no physiological effects, due to its low plasma and its short half-life. On the contrary, recent findings show that this molecule may increase energy expenditure and may also have anti-steatotic activity without producing thyrotoxic effects and according to some authors without affecting the secretion of TSH. The present study carried out on Wistar rats further confirms this effect. However, at a dose level of $70 \mu \mathrm{g} / 100$ $\mathrm{g}$ BW, the molecule suppresses TSH secretion from hypophysis. The reduction of the thyroid hormone levels that might be secondary to the decreased TSH serum levels, may lead to the reduced activity and expression of NIS, thyroid D1, and TPO. These new data support the idea that exogenous 3,5-T2 causes TSH suppression. On the other hand, a molecule that may increase energy expenditure endowed with protective effect on the liver represents an important therapeutic goal for a more effective treatment of obesity and non-alcoholic fatty liver disease. To this aim, some 3,5-T2 agonists have been synthesized. However, their effects on energy expenditure on the liver and on the hypothalamic-pituitary-adrenal axis still remain to be better defined.

\section{References}

1. Jonas W, Lietzow J, Wohlgemuth F, et al. 3,5-Diiodo-L-thyronine (3,5-t2) exerts thyromimetic effects on hypothalamus-pituitarythyroid axis, body composition, and energy metabolism in male diet-induced obese mice. Endocrinology 2015;156:389-99.

2. Lanni A, Moreno M, Lombardi A, Goglia F. Calorigen effect of diiodothyronines in the rat. J Physiol 1996;494:831-7.

3. Antonelli A, Fallahi P, Ferrari SM, et al. 3,5-diiodo-L-thyronine increases resting metabolic rate and reduces body weight without undesirable side effects. J Biol Reg Homeos Ag 2011;25:655-60.

4. Lanni A, Moreno M, Cioffi M, Goglia F. Effect of 3,3'-diiodothyronine and 3,5-diiodothyronine on rat liver oxidative capacity. Mol Cell Endocrinol 1992;86:143-8.

5. Cioffi F, Zambad SP, Chhipa L, et al. TRC150094, a novel functional analog iodothyronines, reduces adiposity by increasing energy expenditure and fatty acid oxidation in rats receiving a high-fat diet. Fed Am Soc Exp Biol 2010;24:3451-61.

6. Padron AS, Neto RA, Pantaleão TU, et al. Administration of 3,5- 
diiodothyronine (3,5-T2) causes central hypothyroidism and stimulates thyroid-sensitive tissues. J Endocrinol 2014;221:415-27.

7. Silvestri E, Cioffi F, Glinni D, et al. Pathways affected by 3,5-diiodoL-thyronine in liver of high fat-fed rats: evidence from two-dimensional electrophoresis, blue-native PAGE, and mass spectrometry. Mol Bio Syst 2010;6:2256-71.

8. Lanni A, Moreno M, Lombardi A, et al. 3,5-Diiodo-L-thyronine powerfully reduces adiposity in rats by increasing the burning of fats. Fed Am Soc Exp Biol 2005;19:1552-4.

9. Horst C, Harneit A, Seitz HJ, Rokos H. 3,5-Di-iodo-L-thyronine suppresses TSH in rats in vivo and in rat pituitary fragments in vitro. J Endocrinol 1995;145:291-7.

10. Mollica MP, Lionetti L, Moreno M, et al. 3,5-diiodo-L-thyronine, by modulating mithocondrial function, reverses hepatic fat accumulation in rats fed a higt-fat diet. J Hepatol 2009;51:363-70.

11. Cimmino M, Mion F, Goglia F, et al. Demostration of in vivo metabolic effects of 3,5-diiodothyronine. J Endocrinol 1996;149:319-25.

12. Moreno M, Silvetri E, De Matteis R, et al. 3,5-Diiodo-L-thyronine prevents high-fat-diet-induced insulin resistance in rat skeletal muscle through metabolic and structural adaptations. Fed Am Soc Exp Biol 2011;25:3312-24.

13. De Lange P, Cioffi F, Silvestri E, et al. (Healthy) ageing: focus on iodothyronines. Int J Mol Sci 2013;14:13873-92.

14. Moreno M, Lombardi A, Beneduce L, et al. Are the effects of T3 on resting metabolic rate in euthyroid rats entirely caused by $\mathrm{T} 3$ itself? Endocrinology 2002;143:504-10.

15. Giammanco M, Cassata G, Cicero L, et al. 3,5-diiodo-L-thyronine induced modification in pituitary thyroid axis in rats fed higt fat diet. A preliminary report. In: Giammanco M, ed. Proceedings of the 86th SIBS National Congress, 24-25 October 2013, Palermo, Italy. J Biol Res 2015;87:5161.

16. Ball SG, Sokolov J, Chin WW. 3,5-Diiodo-l-thyronine (T2) has selective thyromimetic e Vects in vivo and in vitro. J Mol Endocrinol 1997;19:137-47.

17. Williams GR, Bassett JHD. Deiodinases: the balance of thyroid hormone local control of thyroid hormone action: role of type 2 deiodinase. J Endocrinol 2011;209:261-72.

18. Mendoza A, Navarrete-Ramirez P, Hernandez-Puga G, et al. 3,5-T2 is an alternative ligand for the thyroid hormone receptor b1. Endocrinology 2013;154:2948-58.

19. Gereben B, Goncalves C, Harney JW, et al. Selective proteolysis of human type 2 deiodinase: a novel ubiquitin-proteasomal mediated mechanism for regulation of hormone activation. Mol Endocrinol 2000;14:1697-708.

20. Ikuyama S, Ohe K, Takayanagi R, et al. Cloning and characterization of the $4.2 \mathrm{~kb}$ region of the rat thyrotropin receptor promoter. Endocr J 1997;44:247-56.

21. Milenkovic M, De Deken X, Jin L, et al. Duox expression and related H2O2 measurement in mouse thyroid: onset in embryonic development and regulation by TSH in adult. $\mathrm{J}$ Endocrinol 2007;192:615-26.

22. Weyemi U, Caillou B, Talbot M, et al. Intracellular expression of reactive oxygen species-generating NADPH oxidase NOX4 in normal and cancer thyroid tissues. Endocr-Relat Cancer 2010;17:27-37. 